\title{
Latent learning in end stage renal disease (ESRD)
}

Article

Accepted Version

Jones, D. J. W., Butler, L. T., Harris, J. P. and Vaux, E. C. (2015) Latent learning in end stage renal disease (ESRD). Physiology \& Behavior, 142. pp. 42-47. ISSN 0031-9384 doi: https://doi.org/10.1016/j.physbeh.2015.01.033 Available at https://centaur.reading.ac.uk/40261/

It is advisable to refer to the publisher's version if you intend to cite from the work. See Guidance on citing.

Published version at: http://www.sciencedirect.com/science/article/pii/S0031938415000475

To link to this article DOI: http://dx.doi.org/10.1016/j.physbeh.2015.01.033

Publisher: Elsevier

All outputs in CentAUR are protected by Intellectual Property Rights law, including copyright law. Copyright and IPR is retained by the creators or other copyright holders. Terms and conditions for use of this material are defined in the End User Agreement.

\section{www.reading.ac.uk/centaur}

\section{CentAUR}

Central Archive at the University of Reading

Reading's research outputs online 
Daniel J. W. Jones, ${ }^{a}$ Laurie T. Butler, ${ }^{a}$ John P. Harris, ${ }^{a}$ and Emma C Vaux, ${ }^{b}$

${ }^{a}$ School of Psychology and Clinical Language Sciences, University of Reading, Earley Gate, Whiteknights, Reading, Berkshire, RG6 6AL, U.K.

b Department of Renal Medicine, Royal Berkshire NHS Foundation Trust, London Road, Reading, RG1 5AN, U.K.

\section{Corresponding author:}

Daniel J. W. Jones

School of Psychology and Clinical Language Sciences

University of Reading

Earley Gate, Whiteknights

Reading, Berkshire

RG6 6AL

United Kingdom

Tel: +447506724244

Email: d.jones6@ reading.ac.uk 


\begin{abstract}
Cognitive functions such as attention and memory are known to be impaired in End Stage Renal Disease (ESRD), but the sites of the neural changes underlying these impairments are uncertain. Patients and controls took part in a latent learning task, which had previously shown a dissociation between patients with Parkinson's disease and those with medial temporal damage. ESRD patients $(n=24)$ and age and education-matched controls $(n=24)$ were randomly assigned to either an exposed or unexposed condition. In Phase 1 of the task, participants learned that a cue (word) on the back of a schematic head predicted that the subsequently seen face would be smiling. For the exposed (but not unexposed) condition, an additional (irrelevant) colour cue was shown during presentation. In Phase 2, a different association, between colour and facial expression, was learned. Instructions were the same for each phase: participants had to predict whether the subsequently viewed face was going to be happy or sad. No difference in error rate between the groups was found in Phase 1, suggesting that patients and controls learned at a similar rate. However, in Phase 2, a significant interaction was found between group and condition, with exposed controls performing significantly worse than unexposed (therefore demonstrating learned irrelevance). In contrast, exposed patients made a similar number of errors to unexposed in Phase 2. The pattern of results in ESRD was different from that previously found in Parkinson's disease, suggesting a different neural origin.
\end{abstract}

Index words: End stage renal disease (ESRD); Cognitive impairment; Learning; Cortical dysfunction 


\subsection{Introduction}

End Stage Renal Disease (ESRD) occurs when the kidneys (responsible for controlling the water and ionic balance of the body) are functioning at approximately less than $15 \%$ of their normal level. UK estimates suggest that approximately 55,000 patients are currently receiving treatment for ESRD (UK Renal Registry $14^{\text {th }}$ Annual Report, 2013). In addition to the physiological problems associated with the disease, cognition is also known to be impaired in these individuals (Hart \& Kreutzer, 1988). Although the exact pathophysiology of the deficit is not yet fully understood, a number of functions have been found to be impaired. Elias et al. (2009) demonstrated that patients with a lower Glomerular Filtration Rate (GFR) function (standardised measure of kidney functioning) were poorer on tests of visual-spatial memory, working memory, concentration and attention. Lux et al. (2010) suggested that language areas, also located in the temporal cortex, responsible for word fluency and verbal comprehension, may also be impaired in ESRD patients. Other cognitive processes found to be worse in ESRD are planning (Murray, Pederson \& Tupper, 2007), attention and task-switching (Griva, Newman \& Harrison, 2003), and inhibition (Williams, Sklar, Burright \& Donovick, 2004), all usually classified as executive functions and thought to be modulated by the frontal lobes (Stuss \& Knight, 2013; Tranel, Anderson \& Benton, 1994). However, more recently, there have been opposing views in the literature, suggesting that other areas, both cortical and subcortical, may be contributing to such executive tasks in ESRD (Alvarez \& Emory, 2006; Miller \& Cummings, 2007). Duke and Kaszniak (2000) suggested that poorer executive functioning cannot be labelled as a solely "frontal lobe deficit": projections from frontal lobe areas to temporal, parietal and even subcortical areas may be having a modulatory effect during executive functioning tasks. Thus, at present, there is disagreement about the origin of the cognitive dysfunction demonstrated in ESRD.

At present, evidence on the neural changes underlying impairments in ESRD is limited. At this stage, it seems appropriate to initially examine broader distinctions between brain regions, with the expectation that this would allow more targeted investigations in the future. A key distinction often made in relation to other neurological disorders such as Alzheimer's disease (AD) and Parkinson's disease (PD), is that between cortical and subcortical structures. Although the majority of the behavioural data in ESRD suggests a cortical impairment (Kurella, Chertow Luan \& Yaffe, 2004; Lux et al., 2010; Sarnak et al., 2013), there have also been proposals that the pattern of the impairment matches that of a subcortical 
deficit (Harciarek et al., 2010; Pereira et al., 2007). We attempted to distinguish between these possibilities with a dissociation paradigm developed by Myers et al. (2003). Elucidating the relative contributions of different brain regions to the impairments may allow firmer comparisons between ESRD and other neurological disorders.

Myers et al. (2003) showed a dissociation between patients with medial temporal (cortical) and those with basal ganglia (subcortical) damage using a latent learning task. This task was split into two phases. In the first phase, two groups of participants learn to associate one stimulus with another, with one ('exposed') group being exposed to an additional uncorrelated cue. In the second phase, participants learn a new association between stimuli, in which the previously uncorrelated cue (observed by the 'exposed' group) now becomes relevant. Healthy controls exposed to such irrelevant cues in Phase 1 were slower to learn subsequent associations in Phase 2, in which the cue had become relevant, than were controls that had no prior exposure (an effect labelled 'learned irrelevance'). However, patients with medial temporal (MT) damage did not show any effect of exposure in Phase 1: both exposed and non-exposed groups learnt at the same rate. In contrast, patients with basal ganglia damage (PD patients) showed the opposite effect to the controls: patients who were exposed in Phase 1 learnt at a faster rate in Phase 2 than those who were not exposed to the initially irrelevant stimuli. One should be cautious in ascribing the differences between the groups to particular brain areas, since it is difficult to rule out the possibility of more widespread abnormality in the patient groups, an issue which we take up in the Discussion. Nevertheless, at first sight, these three distinct patterns of response suggest dissociable contributions from the medial temporal lobe and basal ganglia to learning and memory. Although the exact mechanisms underlying the different effects are not yet fully understood, they suggest that the task may be useful in identifying cortical and subcortical contributions to impairments in cognitive performance.

We measured the performance of a group of ESRD patients and a matched healthy control group on the test of latent learning. Although the experimental design was the same as in the Myers et al. (2003) study, the stimuli were different, allowing easier production and instruction to participants. We expected to replicate the finding of Myers et al. that healthy controls learn more slowly after prior exposure to the initially uncorrelated stimulus than after no previous exposure (in other words, show 'learned irrelevance'). Because the latent learning task has been run on other patient groups, we can compare the results of ESRD patients with those from other disorders in which the sites of damage are better understood. If 
the changes underlying impairments in ESRD patients are indeed cortical, their latent learning should be more like that of MT than that of PD patients. 


\subsection{Material and methods}

\subsubsection{Participants}

Twenty-four patients (mean age: 67.5 years, S.D: 13.7) were recruited from the renal unit at the Royal Berkshire Hospital, Reading, UK. ESRD patients had been receiving haemodialysis (HD) treatment three times per week for 3-5 hours per treatment, for $>90$ days prior to testing $(\mathrm{Kt} / \mathrm{v}>1.4)$. Patients were deemed eligible for the study by the treating nephrologist who informed them about the study and obtained consent. Patients were excluded if they had any prior history of ophthalmological or neurological illness. Testing was conducted in a quiet office located on the renal ward.

Twenty-four healthy control participants (mean age: 67.8 years, S.D: 13.1) were recruited from a research panel maintained by the University of Reading. Control participants were individually matched to patients on age, sex and education level. Participants were tested in a quiet room in the School of Psychology and Clinical Language Sciences.

\subsubsection{Apparatus \& Materials}

Stimuli were presented on a Toshiba 17.3" LCD screen laptop computer using custom software written in E-Prime (v2.0). The participant was seated in a comfortable chair positioned approximately $45 \mathrm{~cm}$ from the screen. In addition to the primary task, all participants completed the Mini Mental State Examination (MMSE), the Instrumental Activity of Daily Living scale (IADL), the National Adult Reading Test (NART), an estimator of pre-morbid IQ, and the Geriatric Depression Scale (GDS).

\subsubsection{Stimuli}

On each trial, participants were presented with a circle (representing the back of a person's head), with a speech bubble above containing a word printed in black lowercase letters (see Figure 1.1A). The words were taken from the list of 30 monosyllabic non-words used by Myers et al. For each participant, 15 words were randomly selected and used in Phase 1, with the remaining 15 words used in Phase 2 (see Appendix A for word list). In Phase 1 the word "melk" was the signal word, $W$, that predicted a 'happy' face, whereas all the other 14 words predicted a 'sad' face. The circle could be coloured red or green, or uncoloured (white), however, in Phase 1 the colour was unrelated to the happiness of the face. In Phase 2, either 
red or green was selected to be colour $\mathrm{C}+$ which predicted a 'happy' face, whilst the other colour was colour C- which predicted a 'sad' face. Colours were counterbalanced, so that red was colour $\mathrm{C}+$ for half of participants, and green was colour $\mathrm{C}+$ for the other half.

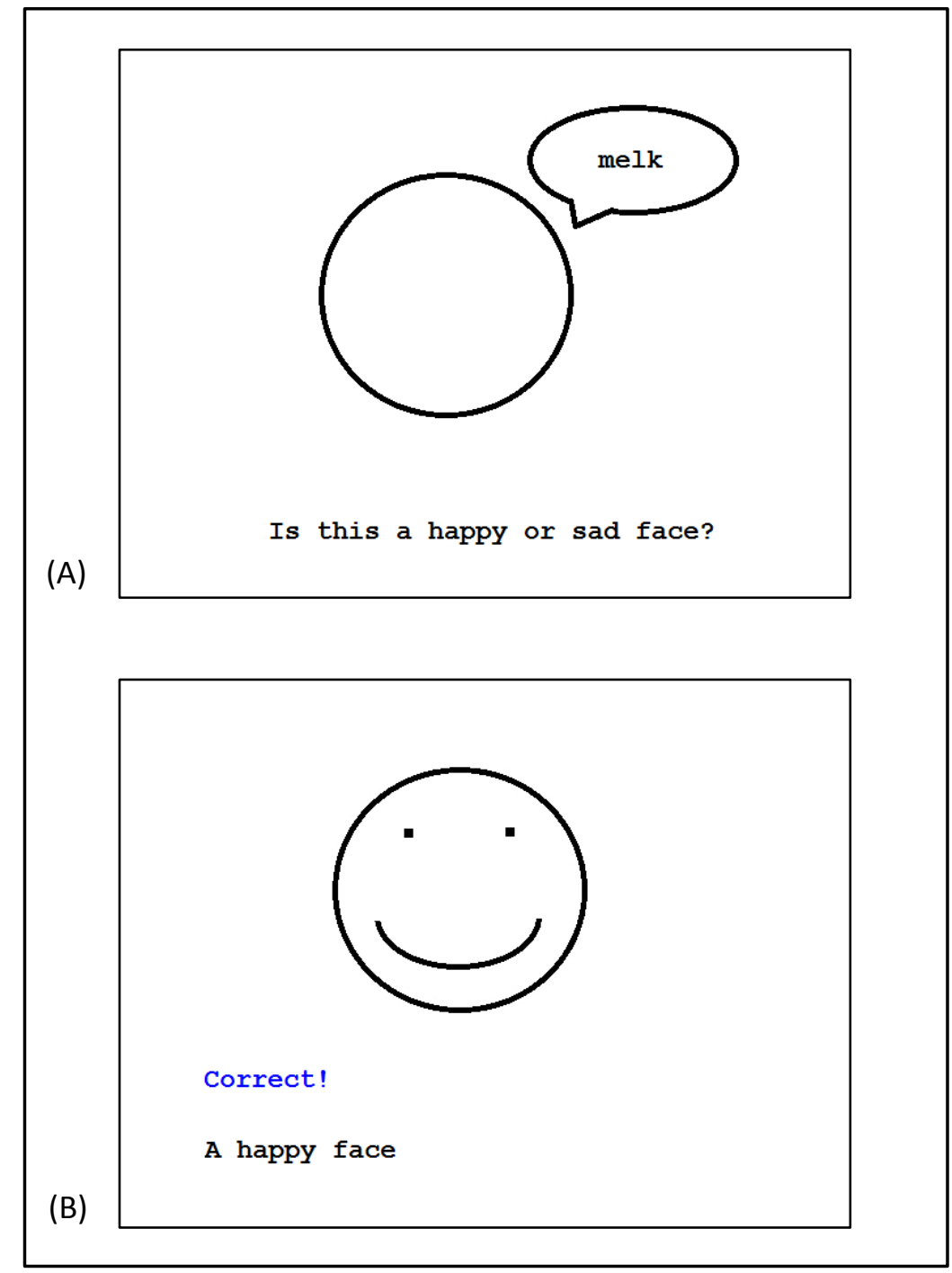

Figure 1.1. Example of the screen appearance at: (A) the start of each trial (B) after the participant responds correctly.

\subsubsection{Design and Procedure}

Participants in both the control and patient groups were randomly assigned to either an exposed $(n=12)$ or unexposed $(n=12)$ group. All participants took part in Phase 1 and Phase 2 of the experiment. 
Before Phase 1 began, participants were told that they would view a series of back views of heads, so the face would not be visible. Their task would be to predict whether the face to be shown would be happy or sad when the head turned around. On each trial, the back of the head, speech bubble and word appeared together, with a question underneath, "Is this a happy or sad face?". The participant responded by pressing one of two keys ('a' for happy, and ' 1 ' for sad). The face (either 'happy' or 'sad') was then shown, together with corrective feedback (see Figure 1.1B).

Phase 1 consisted of 30 trials. During 15 randomly selected trials, the signal word, $W$, ("melk") was presented, and the 'happy' face appeared after the participant had responded. On the remaining 15 trials, the other words were presented in a random order and a 'sad' face appeared. In Phase 1, the signal word, "melk", perfectly predicted the appearance of a 'happy' face. For participants in the Phase 1 exposed group, the head appeared in colour C+ for 15 trials and in colour C- for 15 trials, but colour and facial expression were randomly paired. For participants in the unexposed condition, the head was always uncoloured (white).

Phase 2 began about 1 minute after the end of Phase 1 (the time needed to close one program and download the other). The faces used in Phase 1 were also shown during Phase 2, but in the second phase colour predicted facial expression perfectly. Phase 2 consisted of 60 trials, split into four blocks of 15 trials. Between each block, participants were given a short break. Each of the 15 monosyllabic non-words occurred once in each block, in a randomised order, but the words were related to the subsequent facial expression. In Phase 2, colour $\mathrm{C}+$ occurred randomly on five trials per block, with colour $\mathrm{C}$ - occurring on the remaining 10 trials. Colour $\mathrm{C}+$ was predictive of (correlated with) a subsequent 'happy' face, whereas colour C- was predictive of (correlated with) a 'sad'. In Phase 2, the previously predictive words were now irrelevant to the prediction of facial expression.

In both phases of the task, number of errors was used as the measure of performance. In Phase 2, participants were deemed to have reached learning criterion if 10 consecutive correct responses were made (with consecutive responses across blocks being recognised), whereupon Phase 2 was terminated. Criterion cut off was included to prevent errors occurring due to boredom with the task. 


\subsection{Results}

\subsubsection{Demographics}

Table 1.1 shows participants' means and standard deviations for age, years of education, NART overall IQ, GDS, MMSE and IADL score, split by group and condition. Higher scores reflect better performance, except for the GDS in which a higher score reflects a greater sign of depression. A 2 (patient vs. controls) x 2 (exposed vs. unexposed) between subjects ANOVA found no main effect of group (patients vs controls) for age $(F(1,44)=0.004, p=$ $0.949)$, years of education $(F(1,44)=0.149, p=0.701)$, $\operatorname{MMSE}(F(1,44)=2.521, p=$ $0.120)$, or NART $(F(1,44)=2.452, p=0.125)$. However, a main effect of group was found for $\operatorname{GDS}(F(1,44)=8.686, p=0.005)$ and IADL score $(F(1,44)=18.109, p<0.001)$. No significant main effect was found of condition (exposed vs unexposed) for age $(F(1,44)=$ $0.002, p=0.966)$, years of education $(F(1,44)=0.003, p=0.956), \operatorname{MMSE}(F(1,44)=1.120$, $p=0.296), \operatorname{NART}(F(1,44)=1.318, p=0.257)$ or $\operatorname{IADL}(F(1,44)=0.637, p=0.429)$. However, a significant main effect was found for $\operatorname{GDS}$ score $(F(1,44)=4.503, p=0.04)$. No significant interactions were found involving age $(F(1,44)=0.837, p=0.365)$, years of education $(F(1,44)=0.003, p=0.956), \operatorname{MMSE}(F(1,44)=0.124, p=0.726), \operatorname{NART}(F(1$, $44)=0.338, p=0.564), \operatorname{GDS}(F(1,44)=0.347, p=0.559)$ or $\operatorname{IADL}(F(1,44)=0.637, p=$ $0.429)$.

Although there was a significant difference between patient and control IADL scores, the mean patient score and all individual patient scores were within the normal range. Despite the significant differences in GDS scores, the mean patient GDS score was within the normal range, though the individual scores for 3 patients were consistent with a moderate level of depression. We consider below whether these differences between groups might have influenced the results of the latent learning task. Table 1.2 provides further information on ESRD patients' initial diagnoses, comorbidities and the duration of time that patients have been receiving haemodialysis. 
Table 1.1. Demographic information for patient and control groups - split by condition.

\begin{tabular}{|c|c|c|c|c|}
\hline & \multicolumn{4}{|c|}{ Group (exposed) } \\
\hline & \multicolumn{2}{|c|}{ Patient $(n=12)$} & \multicolumn{2}{|c|}{ Control $(n=12)$} \\
\hline & Mean & SD & Mean & SD \\
\hline Age & 65.8 & 14.6 & 69.7 & 14.3 \\
\hline Education & 11.8 & 2.7 & 12.0 & 2.5 \\
\hline MMSE & 27.8 & 2.1 & 28.3 & 1.1 \\
\hline NART Overall IQ & 114.5 & 6.3 & 119.6 & 5.1 \\
\hline GDS & 4.8 & 2.8 & 2.3 & 1.5 \\
\hline IADL & 6.9 & 1.5 & 8.0 & 0.0 \\
\hline \multirow[t]{4}{*}{$\mathbf{M} / \mathbf{F}$} & 8 & 4 & 7 & 5 \\
\hline & \multicolumn{4}{|c|}{ Group (unexposed) } \\
\hline & \multicolumn{2}{|c|}{ Patient $(n=12)$} & \multicolumn{2}{|c|}{ Control $(n=12)$} \\
\hline & Mean & SD & Mean & SD \\
\hline Age & 69.3 & 13.2 & 65.9 & 11.9 \\
\hline Education & 11.8 & 2.7 & 12.1 & 2.6 \\
\hline MMSE & 28.1 & 2.1 & 29.0 & 1.0 \\
\hline NART Overall IQ & 105.6 & 33.9 & 116.7 & 6.8 \\
\hline GDS & 2.8 & 3.6 & 1.2 & 1.2 \\
\hline IADL & 6.4 & 1.6 & 8.0 & 0.0 \\
\hline $\mathbf{M} / \mathbf{F}$ & 7 & 5 & 8 & 4 \\
\hline
\end{tabular}


Table 1.2. ESRD patient group characteristics

Haemodialysis Patients $(n=24)$

\begin{tabular}{|c|c|c|}
\hline Characteristic & No. of Patients & $\begin{array}{l}\text { Percentage or Mean } \\
\pm \text { SD } \\
\end{array}$ \\
\hline \multicolumn{3}{|l|}{ Age (yr) } \\
\hline$<55$ & 4 & 16.7 \\
\hline $55-64$ & 6 & 25.0 \\
\hline $65-74$ & 5 & 20.8 \\
\hline $75-85$ & 8 & 25.0 \\
\hline$>85$ & 1 & 12.5 \\
\hline Mean & & $67.5 \pm 13.7$ \\
\hline \multicolumn{3}{|l|}{ Dialysis Duration (months) } \\
\hline $0-12$ & 3 & 12.5 \\
\hline $13-24$ & 6 & 25.0 \\
\hline$>24$ & 15 & 62.5 \\
\hline Mean & & $50.6 \pm 60.4$ \\
\hline \multicolumn{3}{|l|}{ Cause of ESRD } \\
\hline Type 2 diabetes mellitus & 3 & 12.5 \\
\hline Type 1 diabetes mellitus & 1 & 4.2 \\
\hline Adult polycystic kidney disease & 4 & 16.7 \\
\hline Chronic kidney disease (unknown cause) & 4 & 16.7 \\
\hline Obstructive uropathy & 2 & 8.3 \\
\hline Glomerulonephritis & 6 & 25.0 \\
\hline Vasculitis & 2 & 8.3 \\
\hline Hypertensive/renovascular disease & 1 & 4.2 \\
\hline Surgical loss & 1 & 4.2 \\
\hline \multicolumn{3}{|l|}{ Comorbid Conditions } \\
\hline Peripheral vascular disease & 7 & 29.2 \\
\hline Diabetes & 8 & 33.3 \\
\hline Hypertension & 17 & 70.8 \\
\hline Stroke & 3 & 12.5 \\
\hline Myocardial infarction & 1 & 4.2 \\
\hline
\end{tabular}




\subsubsection{Patients vs. Controls}

Figure 1.2 shows the mean number of errors during Phase 1 and Phase 2 for both patients and controls. Due to the non-normal nature of the distribution of scores, log transformations were performed to normalise the data to enable parametric tests to be carried out (raw scores will still be presented throughout to show mean differences). A 2 (patient vs control) x 2 (exposed vs unexposed) x 2 (Phase 1 vs Phase 2) between subjects ANOVA showed a main effect of group, $F(1,88)=5.246, p=0.024$, and condition $F(1,88)=18.75, p<0.001$, but no main effect of phase, $F(1,88)=0.069, p=0.794$. The only significant interaction found was between group and condition, $F(1,88)=4.608, p=0.035$. All other interactions were nonsignificant: group $\mathrm{x}$ phase, $F(1,88)=0.008, p=0.930$, condition $\mathrm{x}$ phase, $F(1,88)=1.134$, $p=0.290$, and group $\mathrm{x}$ condition $\mathrm{x}$ phase, $F(1,88)=2.459, p=0.120$. Removing the 3 patients with elevated GDS scores did not alter the pattern of significance of the main effects or interactions, and so their data were retained. In subsequent analyses, Phase 1 and Phase 2 were analysed separately to determine exactly what was driving the main effect of group and condition (as in Myers et al., 2003). GDS and IADL scores were included in the analyses as covariates, because of the inter-group differences in these variables. Because of the wide range of ages of participants, and the possibility that age was affecting latent learning, age was also included as a covariate. For Phase 1 there was a main effect of group, $F(1,41)=$ $7.109, p=0.011$, and condition, $F(1,41)=7.134, p=0.011$. However, there was no significant interaction found between group $\mathrm{x}$ condition, $F(1,41)=0.404, p=0.529$, suggesting that patients were performing similarly to controls during Phase 1. None of the covariates had a significant effect (for age, $\mathrm{F}(1,41)=1.415, p=0.241$, for IADL, $\mathrm{F}(1,41)=$ $0.686, p=0.412$, and for GDS, $\mathrm{F}(1,41)=0.541, p=0.466)$. For Phase 2 , there was no main effect of group, $F(1,41)=0.341, p=0.563$, but there was a main effect of condition, $F(1,41)$ $=6.733, p=0.013$, and a significant group $\mathrm{x}$ condition interaction, $F(1,41)=4.986, p=.031$. As in Phase 1, none of the covariates had a significant effect (for age, $\mathrm{F}(1,41)=0.467, p=$ 0.498 , for IADL, $\mathrm{F}(1,41)=0.086, p=0.77$, and for $\mathrm{GDS}, \mathrm{F}(1,41)=1.112, p=0.298)$. Independent t-tests, with Bonferroni correction, revealed controls to be making significantly fewer errors during the un-exposed condition $(M=5.67, S D=9.88)$ than the exposed condition $(M=18.58, S D=11.20)$ during Phase $2(t(22)=4.01, p=.004)$. In contrast, the patient un-exposed $(M=14.17, S D=12.47)$ and exposed group $(M=16.50, S D=12.21)$ did not differ significantly during Phase $2(t(22)=0.608, p>0.9)$. An exposure effect observed in the control group is not apparent in the patient group. 


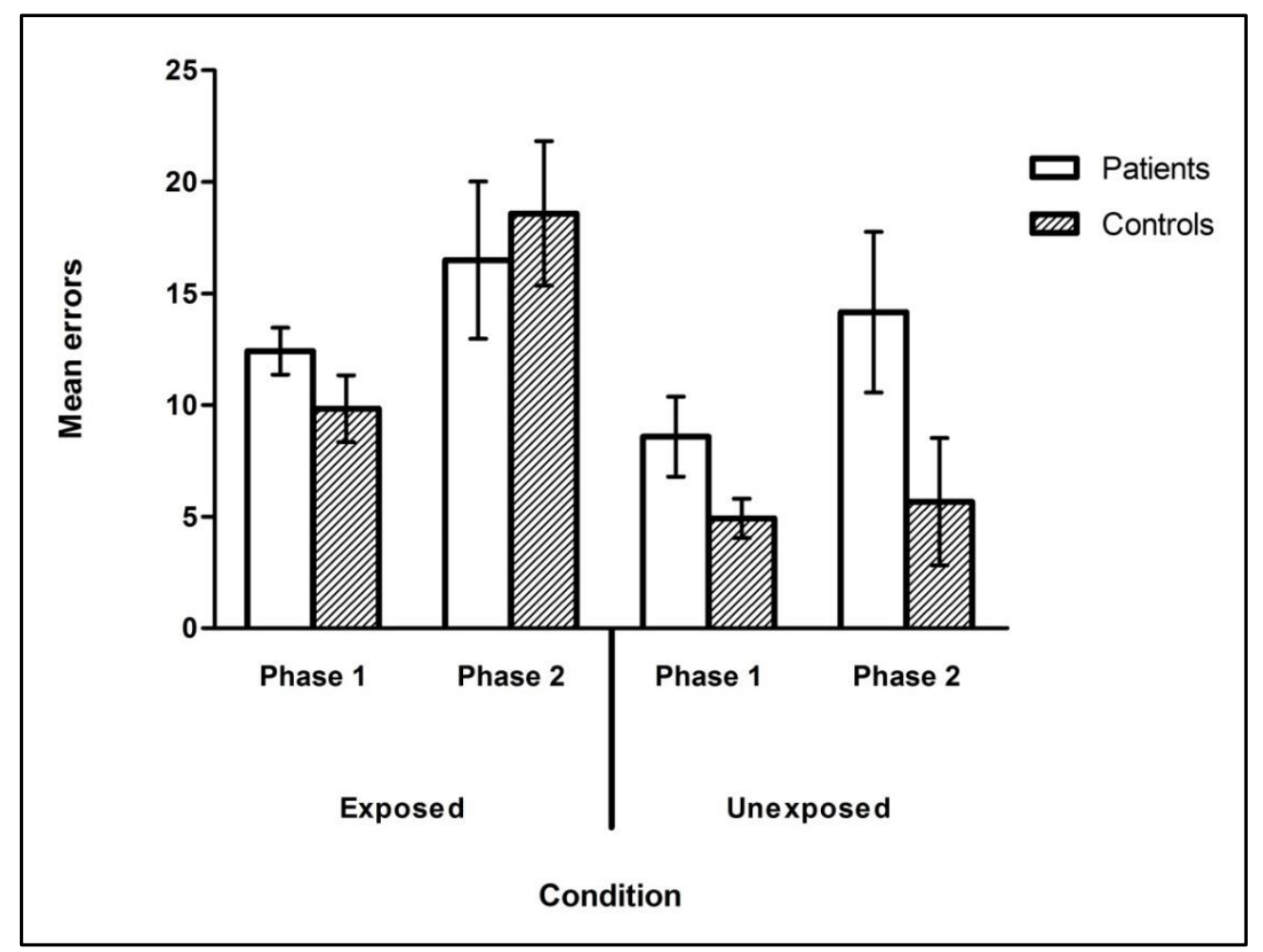

Figure 1.2. Total number of errors made during Phase 1 and Phase 2 for both patients and controls in each condition. Error bars $= \pm 1$ S.E.

Figure 1.3 shows the percentage of participants who reached criterion during Phase 2. To determine if participants were reaching criterion differently, dependent on condition, Chisquared tests were carried out. In the control group, there was no significant difference between the number of participants who reached criterion in the un-exposed condition and the exposed condition $\left(\chi^{2}(1)=3.00, p=.083\right)$. In the patient group, there was absolutely no difference between the number of participants reaching criterion in the un-exposed condition compared to the exposed condition $\left(\chi^{2}(1)=0.00, p=1.0\right)$. Irrespective of condition, patients reached criterion exactly the same number of times.

To summarise, patients were responding in a similar way to controls during Phase 1 in each condition, however, during Phase 2, the exposure effect observed in the control group was not evident in the patient group: exposure condition did not impact on patient error rate. 


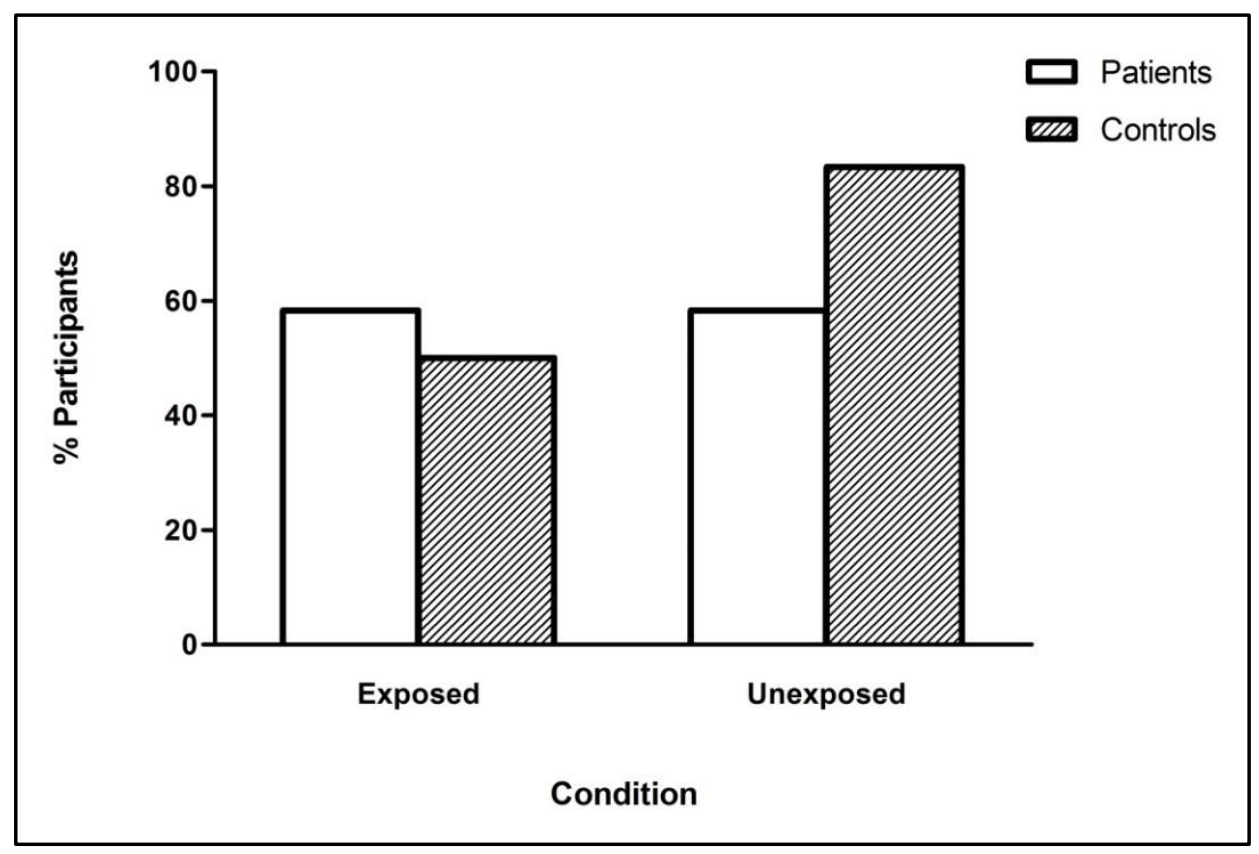

Figure 1.3. Total percentage of participants reaching criterion in Phase 2.

\subsubsection{Correlations}

The absence of learned irrelevance in the ESRD group suggests that patients are processing the stimuli in a qualitatively different way. However, due to the lower IADL score and, more importantly, the higher GDS score observed in the ESRD group, it is possible that the impairment abolishing learned irrelevance in the latent learning task is a consequence of patients' general cognitive status rather than an effect of ESRD. For example, high depression levels have been associated with impaired verbal and non-verbal learning, concentration, attention and retention (for a review see Veiel, 1997). To investigate this possibility, Spearman correlations were calculated between scores on Phase 2 of the latent learning task (in which patients and controls differed) and age, education level, MMSE, NART, GDS and IADL score. Correlations were calculated separately for the patient and control group and split by condition (exposed vs unexposed). See Table 1.3 for correlations and p-values. None of the Spearman correlations was significant, suggesting that the major factor driving this difference is ESRD.

The correlations between age and errors in Phase 2, though insignificant, were negative in patients and positive in controls in both the exposed and unexposed groups. When the scores 
from the exposed and unexposed groups were combined, the correlations were still insignificant (for patients, $r=0.28, p=0.19$; for controls, $r=0.30, p=0.16$ ).

Table 1.3. Spearman correlation coefficients ( $p$-value in brackets) between Phase 2 mean errors and measures of cognition and mood (split by condition). No significant correlations were found.

\section{Patient Group}

\begin{tabular}{|c|c|c|c|c|c|c|}
\hline & Age & Education & MMSE & NART & IADL & GDS \\
\hline \multirow{3}{*}{ Exposed } & -.539 & .055 & .570 & -.088 & .261 & $(-.278)$ \\
\hline & & & & & & \\
\hline & $(.071)$ & $(.866)$ & $(.053)$ & $(.786)$ & $(.412)$ & .382 \\
\hline \multirow{3}{*}{ Unexposed } & -.170 & -.250 & .257 & -.209 & $* * *$ & -.373 \\
\hline & & & & & & \\
\hline & $(.598)$ & $(.434)$ & $(.421)$ & $(.515)$ & $* * *$ & $(.232)$ \\
\hline
\end{tabular}

\section{Control group}

\begin{tabular}{lllllll}
\hline & Age & Education & MMSE & NART & IADL & GDS \\
\cline { 2 - 7 } Exposed & .467 & -.075 & .329 & .161 & -.169 & .106 \\
& $(.126)$ & $(.817)$ & $(.297)$ & $(.616)$ & $(.600)$ & $(.742)$ \\
& .390 & -.179 & -.311 & -.215 & $* * *$ & .344 \\
Unexposed & $(.210)$ & $(.578)$ & $(.326)$ & $(.502)$ & $* * *$ & $(.274)$ \\
& & & & & & \\
\hline
\end{tabular}

MMSE = Mini Mental State Examination, NART = National Adult Reading Test, IADL = Instrumental Activities of Daily Living, GDS = Geriatric Depression Score. Two-tailed correlations were calculated separately for each group. *** $=$ correlation could not be conducted due to lack of variance between scores, with all controls and patients in the unexposed groups achieving the maximum score (within the exposed groups, all IADL scores were within the normal range). 


\subsubsection{Possible effect of hypertension}

As Table 1.2 shows, ESRD is often associated with comorbidities. In the present patient group, one comorbidity, namely hypertension, was present in sufficiently large numbers to make a comparison possible between patients with and without this symptom. However, no main effect of hypertension was found for the data in Phase $1(F(1,20)=1.542, p=0.229)$, or in Phase $2(F(1,20)=0.044, p=0.836)$. In addition, no significant interaction between hypertension and condition was found in either Phase $1(F(1,20)=0.921, p=0.349)$ or Phase $2(F(1,20)=0.642, p=0.432)$.

\subsection{Discussion}

This study aimed to advance knowledge of the nature of the deficit underlying cognitive impairments in ESRD. To investigate this, a latent learning task, previously showing a dissociation between patients with medial temporal and basal ganglia damage, was employed. In line with Myers et al. (2003), controls showed an exposure effect: learning was slower in the exposed compared to the un-exposed condition. These findings mimic the results of studies investigating the effect of learned irrelevance (Dess \& Overmier, 1989; Maes, Dame $\&$ Eling, 2004). Baker (1976) suggests that due to the unfamiliar setting, an expectation arises that two cues are unrelated to one another which perseverates into subsequent phases of the task, resulting in slower learning when stimuli do become relevant.

In the ESRD group, patients learned at the same rate, irrespective of exposure: the number of errors in the exposed group was the same as that in the un-exposed group. Patients' overall learning rate, in both conditions, was similar to the rate of controls' exposed learning rate. The main difference between the control and the patient groups was in how they responded during the un-exposed condition, since novel prior exposure did not influence patient learning rate.

Although there was a similar lack of effect of exposure in our ESRD group and the medial temporal group in the Myers et al. (2003) study, the absolute levels of learning in Phase 2 are very different. The MT patients of Myers et al. not only learned in un-exposed Phase 2 as quickly as controls, but they also learned in exposed Phase 2 more quickly than controls, with fewer errors. In our ESRD group, the patients learned at the same rate as the exposed controls 
(a similar number of errors was made), but learned at a slower rate than the un-exposed controls (making about 2.5 times more errors, though the difference was not significant after Bonferroni correction). Myers et al. suggest that medial temporal damage is facilitating this simple stimulus-response learning because of reduced interference from the damaged hippocampus (animal studies have shown that lesions to the hippocampal region result in faster stimulus-response learning [Eichanbaum, Otto \& Cohen, 1994]). However, we found no evidence for any such facilitation in our ESRD group. In their PD patients, Myers et al found a 'reversed' exposure effect. That is, in Phase 2, their unexposed group made many more errors than did the exposed group, unlike the pattern in ESRD, in which our unexposed patient group made slightly fewer errors than the exposed group. Thus, although the pattern of results in our controls was similar to that of Myers et al., the results of our ESRD group differed from those of both their MT and their PD patients.

One explanation for the pattern found in our ESRD group is that, rather than a facilitation of learning, as observed in MT patients, simple forgetting may be responsible - patients are less able to recall the stimulus information present in Phase 1. Bouten (1993) uses the term 'proactive interference' to describe the learned irrelevance demonstrated in the control group, in which the negative influence from observing the uncorrelated stimulus in Phase 1 is influencing performance in Phase 2. In ESRD, on the other hand, there is no interference between the two phases, as what has been observed during Phase 1 is simply forgotten. Examples of accelerated forgetting have been demonstrated in Alzheimer's patients with temporal lobe pathology (Christensen, Kopelman, Stanhope, Lorentz \& Owen, 1998; Hart, Kwentus, Taylor \& Harkins, 1987) and patients with unilateral and bilateral hippocampal lesions, who have shown immediate (Parkin \& Leng, 1988) and one minute delayed accelerated forgetting (Frisk \& Milner, 1990). Kopelman and Stanhope (1997) demonstrated accelerated forgetting in amnesia patients with temporal lobe damage. During free recall of pictures of objects, greater accelerated forgetting was observed in the temporal lobe patients, compared to a control group, between periods of 20 seconds and 10 minutes. This hypothesis may explain the pattern of results in our ESRD group. Patients are processing Phase 1 correctly, in the same way as controls, but between Phase 1 and Phase 2, stimuli observed in Phase 1 , stored by the control group for future use, are simply forgotten in the patient group. There is evidence for impairment of short-term memory in ESRD. Kurella et al (2004) found ESRD patients to be significantly worse on the California Verbal Learning Test. Similarly, Elias et al (2009) found patients with low kidney function to be worse on tests of immediate 
visual memory than were those whose kidney function was less impaired. These results, and our own, suggest impairment of temporal lobe processing in the illness.

Another possibility is that patients may be processing Phase 1 in a qualitatively different way to the controls (in other words, the exposed group may be ignoring the colours in Phase 1), so that there is no stored information to affect subsequent processing in Phase 2. However, at this stage it is not clear whether this is due to limitations on attentional resources (and so perhaps would implicate the frontal lobes) or some other factor.

The ESRD results are certainly consistent with a cortical impairment of some kind, perhaps involving the frontal and/or temporal lobes. Ruling out an important sub-cortical contribution depends in the present context on a comparison with the results of the PD patients of Myers et al. A limitation of that study, as the authors point out, is that the patients were all receiving dopaminergic medication, and a dose which alleviated basal ganglia impairment (at least partially) might cause hyperactivity of dopaminergic innervation of the frontal lobes. In healthy volunteers, a dopamine agonist (amphetamine) can interfere with latent inhibition (Gray, Pickering, Hemsley, Dawling, and Gray, 1992). However, the effect was found to be dose dependent, so that, somewhat counterintuitively, a $10 \mathrm{mg}$ dose had no effect whereas a 5 $\mathrm{mg}$ dose did. Nevertheless, it is possible that at least some of the PD patients in the Myers et al study fell in the appropriate dose range to cause changes to the frontal lobes. Thus there is uncertainty about whether the pattern of results in the PD patients of Myers et al. reflects frontal or subcortical changes (or both). Since the pattern of results in our ESRD patients is different from that in the medicated PD patients, and there is evidence for temporal impairment in ESRD from studies of immediate memory, we suggest that absence of latent learning in ESRD is most consistent with a temporal lobe impairment. However, without further evidence, we cannot be sure whether the whole cortex is affected or whether some areas are more susceptible than others. Similarly, we do not assume that subcortical structures are immune to the effects of ESRD, since their blood supply will be affected in the same ways as that to the cortex. It may be that subcortical processing is more robust, and so less prone to impairment caused by degradation of the blood.

We should note a limitation of the study. ESRD is linked, by cause or effect, to several comorbidities (see Table 1.2), which also affect blood supply. Our sample of patients contained enough with a diagnosis of hypertension to make possible a comparison of those with and without hypertension. No effect of hypertension was found, perhaps because, once 
diagnosed, it can be controlled by medication. We cannot rule out the possibility that at least some contribution to the impairments we found may be caused by other comorbidities. Further studies are needed to disentangle these possible effects on cognition.

The trend of a rise in number of errors with age in controls is understandable, but the opposite trend in patients is puzzling. There was little variation in severity of illness in this patient group - by definition, anyone in the end-stage of the disease has kidney function of approximately less than $15 \%$ of normal (GFR $<15 \mathrm{~mL} / \mathrm{min}$ ). It is possible that older brains are less susceptible to the changes in blood chemistry caused by ESRD than are younger brains, a possibility which needs investigation in future studies.

In summary, this study provides an insight into the mechanisms underlying the cognitive impairment observed in ESRD, suggesting the deficit to be predominantly cortical in nature, and implicating the involvement of the temporal lobes. Further investigations building on the present results may shed more light on the possible contribution from temporal regions of the brain, perhaps with the aid of brain imaging techniques. 


\section{Acknowledgements}

D. J. W. Jones was supported by an ESRC CASE Research Studentship during the course of this research. We thank the staff of the Royal Berkshire NHS Foundation Trust for their cooperation throughout this research, all our participants for their time and commitment to the project, Dr Jared Smith for drawing the study by Myers et al. to our attention, and an anonymous reviewer for helpful suggestions. 


\section{References}

Alvarez, J. A., \& Emory, E. (2006). Executive function and the frontal lobes: a meta-analytic review. Neuropsychology Review, 16(1), 17-42. doi: 10.1007/s11065-006-9002-x

Baker, A. G. (1976). Learned irrelevance and learned helplessness: Rats learn that stimuli, reinforcers, and responses are uncorrelated. Journal of Experimental Psychology: Animal Behavior Processes, 2, 130-141. doi: 10.1037/0097-7403.2.2.130

Bouton, M. E. (1993). Context, time and memory retrieval in the interference paradigms of pavlovian learning. Psychological Bulletin, 114(1), 80-99. doi: 10.1037/00332909.114.1.80

Christensen, H., Kopelman, M. D., Stanhope, N., Lorentz, L., \& Owen, L. (1998). Rates of forgetting in Alzheimer dementia. Neuropsychologia, 36(6), 547-557. doi: 10.1016/S0028-3932(97)00116-4

Duke, L. M., \& Kaszniak, A. W. (2000). Executive functions in degenerative dementias: A comparative review. Neuropsychology Review, 10, 75-99. doi: 10.1023/A: 1009096603879

Dess, N. K., \& Overmier, J. B. (1989). General learned irrelevance: Proactive effects on Pavlovian conditioning in dogs. Learning and Motivation, 20(1), 1-14. doi: $10.1016 / 0023-9690(89) 90028-3$

Eichanbaum, H., Otto, T., \& Cohen, N. (1994). Two functional components of the hippocampal memory system. Behavioural and Brain Sciences, 17, 449-518. doi: $10.1017 / \mathrm{S} 0140525 \mathrm{X} 00035391$

Elias, M. F., Elias, P. K., Seliger, S. L., Narsipur, S. S., Dore, G. A., \& Robbins, M. A. (2009). Chronic kidney disease, creatinine and cognitive functioning. Nephrology Dialysis Transplantation, 24, 2446-2452. doi: 10.1093/ndt/gfp107 
Frisk, V., \& Milner, B. (1990). The role of the left hippocampal region in the acquisition and retention of story content. Neuropsychologia, 28, 349-359. doi: 10.1016/00283932(90)90061-R

Gray, N.S., Pickering A.D., Hemsley D.R., Dawling S., and Gray J.A. (1992). Abolition of latent inhibition by a single $5 \mathrm{mg}$ dose of d-amphetamine in man. Psychopharmacology, 107, 10, 425-430. doi: 10.1007/BF02245170

Griva, K., Newman, S. P., Harrison, M. J., Hankins, M., Davenport, A., Hansraj, S., \& Thompson, D. (2003). Acute neuropsychological changes in hemodialysis and peritoneal dialysis patients. Health Psychology, 22, 570-578. doi: 10.1037/02786133.22.6.570

Harciarek, M., Williamson, J. B., Biedunkiewicz, B., Lichodziejewska-Niemierko, M., Debska-Slizien, A., \& Rutkowski, B. (2010). Memory performance in adequately dialyzed patients with end-stage renal disease: Is there an association with coronary artery bypass grafting? Journal of Clinical and Experimental Neuropsychology, 32 (8), 881-889. doi: 10.1080/13803391003596454

Hart, R. P., \& Kreutzer, J. S. (1988). Renal system. In R. E. Tarter, D. H Van Thiel, \& K. L. Edwards (Eds.), Medical neuropsychology: The impact of disease on behaviour (pp.99-120). New York: Plenum Press.

Hart, R. P., Kwentus, J. A., Taylor, J. R., \& Harkins, S. W. (1987). Rate of forgetting in dementia and depression. Journal of Consulting and Clinical Psychology, 55, 101105. doi: 10.1037/0022-006X.55.1.101

Kopelman, M. D., \& Stanhope, N. (1997). Rates of forgetting in organic amnesia following temporal lobe, diencephalic, or frontal lobe lesions. Neuropsychology, 11(3), 343-356. doi: 10.1037/0894-4105.11.3.343

Kurella, M., Chertow, G. M., Luan, J., \& Yaffe, K. (2004). Cognitive impairment in chronic kidney disease. American Geriatrics Society, 52, 1863-1869. doi: 10.1111/j.15325415.2004.52508.x 
Lux, S., Mirzazade, S., Kuzmanovic, B., Plewan, T., Eickhoff, S. B., Shah, N. J., Floege, J., Fink, G. R., \& Eitner, F. (2010). Differential activation of memory-relevant brain regions during a dialysis cycle. Kidney International, 78, 794-802. doi: 10.1038/ki.2010.253

Maes, J. H. R., Dame, M. D. C., \& Eling, P. A. T. M. (2004). More learned irrelevance than perseveration errors in rule shifting in healthy subjects. Brain and Cognition, 54(3), 201-211. doi: 10.1016/j.bandc.2004.01.003

Miller, B. L., \& Cummings, J. L. (Eds.). (2007). The human frontal lobes: Functions and disorders. New York: Guilford Press.

Murray, A. M., Pederson, S. L., Tupper, D. E., Hochhalter, A. K., Miller, W. A., Qi Li, M. S., Zaun, D., Collins, A. J., Kane, R., \& Foley, R. N. (2007). Acute variation in cognitive function in hemodialysis patients: A cohort study with repeated measures. American Journal of Kidney Diseases, 50(2), 270-278. doi: 10.1053/j.ajkd.2007.05.010

Myers, C. E., Shohamy, D., Gluck, M. A., Grossman, S., Onlaor, S., \& Kapur, N. (2003). Dissociating medial temporal and basal ganglia memory systems with a latent learning task. Neuropsychologia, 41, 1919-1928. doi: 10.1016/S0028-3932(03)001271

Parkin, A. J., \& Leng, N. R. C. (1988). Comparative studies of human amnesia: Syndrome or syndromes? In H. Markowitsch (Ed.), Information processing by the brain (pp. 107123). Toronto: Hans Huber.

Pereira, A. A., Weiner, D. E., Scott, T., Chandra, P., Bluestein, R., Griffith, J., \& Sarnak, M. J. (2007). Subcortical cognitive impairment in dialysis patients. Hemodialysis International, 11(3), 309-314. doi: 10.1111/j.1542-4758.2007.00185.x 
Sarnak, M. J., Tighiouart, H., Scott, T. M., Lou, K. V., Sorenson, E. P., Giang, L. M., Drew, D. A., Shaffi, K., Strom, J. A., Singh, A. K., \& Weiner, D. E. (2013). Frequency of and risk factors for poor cognitive performance in hemodialysis patients. Neurology, 80, 471-480. doi: 10.1212/WNL.0b013e31827f0f7f

Stuss, D. T., \& Knight, R. T. (Eds.). (2013). Principles of frontal lobe function. Oxford University Press.

The Renal Association. (2013). UK Renal Registry $16^{\text {th }}$ Annual Report.

Tranel, D., Anderson, S. W., \& Benton, A. (1994). Development of the concept of "executive function" and its relationship to the frontal lobes. Handbook of Neuropsychology, 9, 125-148.

Veiel, H. O. F. (1997). A preliminary profile of neuropsychological deficits associated with major depression. Journal of Clinical and Experimental Neuropsychology, 19(4), 587603. doi: 10.1080/01688639708403745

Williams, M. A., Sklar, A. H., Burright, R. G., \& Donovick, P. J. (2004). Temporal effects of dialysis on cognitive functioning in patients with ESRD. American Journal of Kidney Diseases, 43(4), 705-711. doi: 10.1053/j.ajkd.2003.12.031

Zola, S., \& Mahut, H. (1973). Paradoxical facilitation of object reversal learning after transection of the fornix in monkeys. Neuropsychologia, 11, 271-284. doi: $10.1016 / 0028-3932(73) 90038-9$ 


\section{Appendix A - Word Lists}

The following pronounceable nonwords were used in Phase 1:

$\begin{array}{ll}\text { melk (signal word) } & \text { zoch } \\ \text { tawe } & \text { jant } \\ \text { bije } & \text { hund } \\ \text { morv } & \text { sarn }\end{array}$

ratch

zare

moel

slar

malp

cort

slig

The following pronounceable nonwords were used in Phase 2:

$\begin{array}{lc}\text { noge } & \text { glep } \\ \text { juff } & \text { zoyn } \\ \text { frod } & \text { bola } \\ \text { forl } & \text { hewl }\end{array}$

hoor

fion

blae

dett

gwan

vair

rhyl 